\title{
The CALorimetric Electron Telescope (CALET) for high-energy astroparticle physics on the International Space Station
}

\begin{abstract}
O. Adriani ${ }^{26}$, Y. Akaike ${ }^{7}$, K. Asano ${ }^{24}$, Y. Asaoka ${ }^{31}$, M.G. Bagliesi ${ }^{30}$, G. Bigongiari ${ }^{30}$, W.R. Binns ${ }^{32}$, S. Bonechi ${ }^{30}$, M. Bongii ${ }^{26}$, J.H. Buckley ${ }^{32}$, G. Castellini ${ }^{26}$, M.L. Cherry ${ }^{12}$, G. Collazuol ${ }^{27}$, K. Ebisawa ${ }^{9}$, V. Di Felice ${ }^{29}$, H. Fuke ${ }^{9}$, T.G. Guzik ${ }^{12}$, T. Hams ${ }^{3}$, M. Hareyama ${ }^{22}$, N. Hasebe ${ }^{31}$, K. Hibino ${ }^{10}$, M. Ichimura ${ }^{4}$, K. loka ${ }^{11}$, M.H. Israel ${ }^{32}$, A. Javaid ${ }^{12}$, E. Kamioka ${ }^{20}$, K. Kasahara ${ }^{31}$, J. Kataoka ${ }^{31}$, R. Kataoka ${ }^{15}$, Y. Katayose ${ }^{33}$, N. Kawanaka ${ }^{23}$, H. Kitamura ${ }^{16}$, T. Kotani ${ }^{31}$, H.S. Krawczynski ${ }^{32}$, J.F. Krizmanic ${ }^{2}$, A. Kubota ${ }^{20}$, S. Kuramata ${ }^{4}$, T. Lomtadze ${ }^{28}$, P. Maestro ${ }^{30}$, L. Marcelli 29 , P.S. Marrocchesi ${ }^{30}$, J.W. Mitchell ${ }^{14}$, S. Miyake ${ }^{5}$, K. Mizutani ${ }^{19}$, A.A. Moiseev ${ }^{3}$, K. Mori ${ }^{9,31}$, M. Mori ${ }^{18}$, N. Mori ${ }^{26}$, H.M. Motz ${ }^{31}$, K. Munakata ${ }^{21}$, H. Murakami ${ }^{31}$, Y.E. Nakagawa ${ }^{9}$, S. Nakahira ${ }^{9}$, J. Nishimura ${ }^{9}$, S. Okuno ${ }^{10}$, J.F. Ormes ${ }^{25}$, S. Ozawa ${ }^{31}$, F. Palma ${ }^{29}$, P. Papini' ${ }^{26}$, B.F. Rauch ${ }^{32}$, S.B. Ricciarini ${ }^{26, a}$, T. Sakamoto ${ }^{1}$, M. Sasaki ${ }^{3}$, M. Shibata ${ }^{33}$, Y. Shimizu ${ }^{8}$, A. Shiomi ${ }^{17}$, R. Sparvoli ${ }^{29}$, P. Spillantini ${ }^{26}$, I. Takahashi ${ }^{1}$, M. Takayanagi ${ }^{9}$, M. Takita ${ }^{7}$, T. Tamura ${ }^{8,10}$, N. Tateyama ${ }^{10}$, T. Terasawa ${ }^{7}$, H. Tomida ${ }^{9}$, S. Torii ${ }^{8,31}$, Y. Tunesada ${ }^{24}$, Y. Uchihori ${ }^{16}$, S. Ueno ${ }^{9}$, E. Vannuccini ${ }^{26}$, J.P. Wefel ${ }^{12}$, K. Yamaoka ${ }^{13}$, S. Yanagita ${ }^{6}$, A. Yoshida ${ }^{1}$, K. Yoshida ${ }^{20}$, and T. Yuda ${ }^{7}$
\end{abstract}

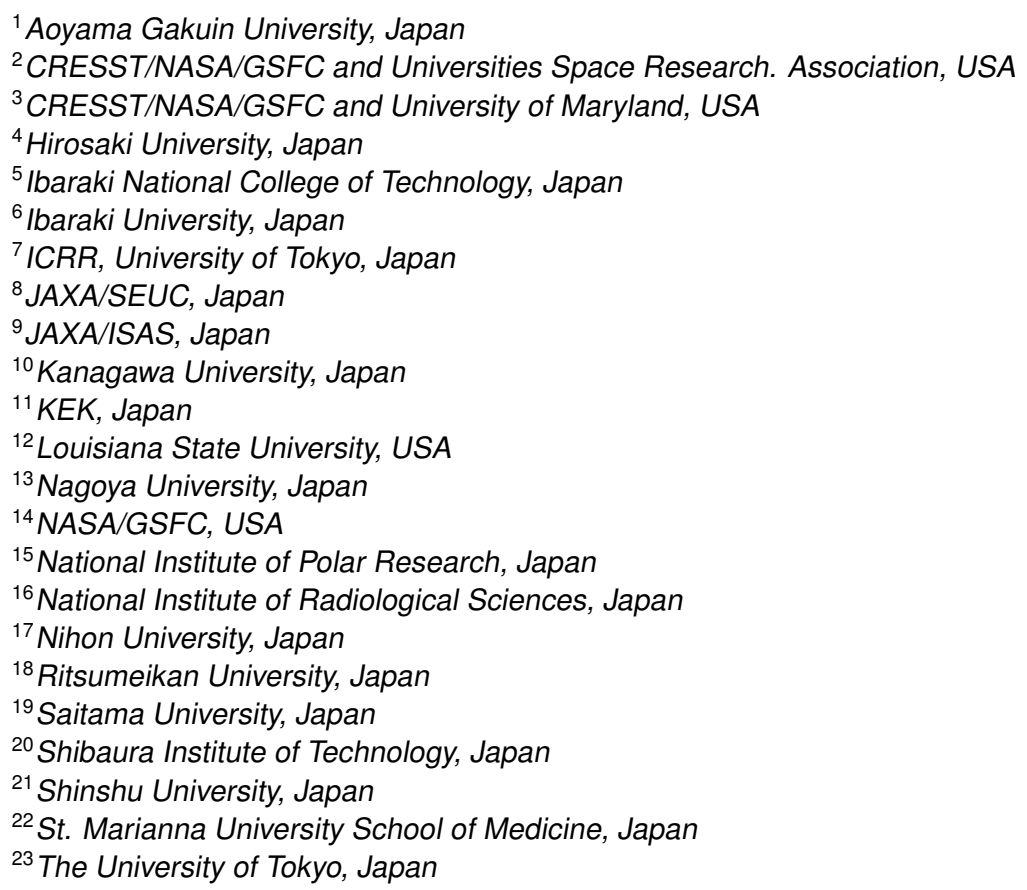

${ }^{\mathrm{a} C}$ Corresponding author, e-mail: s.ricciarini@ifac.cnr.it 


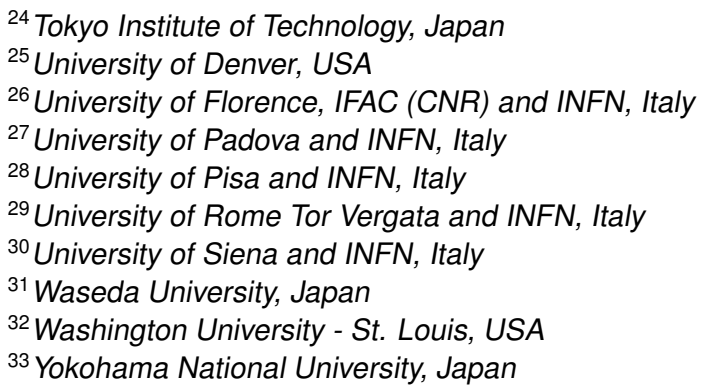

\begin{abstract}
The CALorimetric Electron Telescope (CALET) is a space experiment, currently under development by Japan in collaboration with Italy and the United States, which will measure the flux of cosmic-ray electrons (and positrons) up to $20 \mathrm{TeV}$ energy, of gamma rays up to $10 \mathrm{TeV}$, of nuclei with $\mathrm{Z}$ from 1 to 40 up to $1 \mathrm{PeV}$ energy, and will detect gamma-ray bursts in the $7 \mathrm{keV}$ to $20 \mathrm{MeV}$ energy range during a 5 year mission. These measurements are essential to investigate possible nearby astrophysical sources of high energy electrons, study the details of galactic particle propagation and search for dark matter signatures. The main detector of CALET, the Calorimeter, consists of a module to identify the particle charge, followed by a thin imaging calorimeter ( 3 radiation lengths) with tungsten plates interleaving scintillating fibre planes, and a thick energy measuring calorimeter (27 radiation lengths) composed of lead tungstate logs. The Calorimeter has the depth, imaging capabilities and energy resolution necessary for excellent separation between hadrons, electrons and gamma rays. The instrument is currently being prepared for launch (expected in 2015) to the International Space Station ISS, for installation on the Japanese Experiment Module - Exposure Facility (JEM-EF).
\end{abstract}

\title{
1 Introduction
}

The CALorimetric Electron Telescope CALET [1] [2] will be installed on the International Space Station (ISS) in 2015, with the aim of performing accurate and long-duration observations of highenergy cosmic radiation, including charged particles and photons.

An overview of the CALET mission and apparatus is given in Sect. 2, while Sect. 3 contains a selection of major science items which will be addressed by CALET.

\section{Overview of CALET experiment}

The CALET apparatus is currently in advanced integration and test phase and scheduled to be launched in space in 2015 with the Japanese rocket HTV-5, for installation on the Japanese Experiment Module - Exposure Facility (JEM-EF) on the ISS and subsequent operation for a target duration of 5 years.

The CALET payload (see figure 1) includes scientific and auxiliary equipment for a total mass of $650 \mathrm{~kg}$, dimensions of $1.9 \times 0.8 \times 1.0 \mathrm{~m}^{3}$, nominal power consumption of $650 \mathrm{~W}$. The main scientific instruments are the electromagnetic calorimeter (CALET-CAL, see Sect. 2.1) and the gamma-ray burst monitor (CGBM, see Sect. 2.2). 


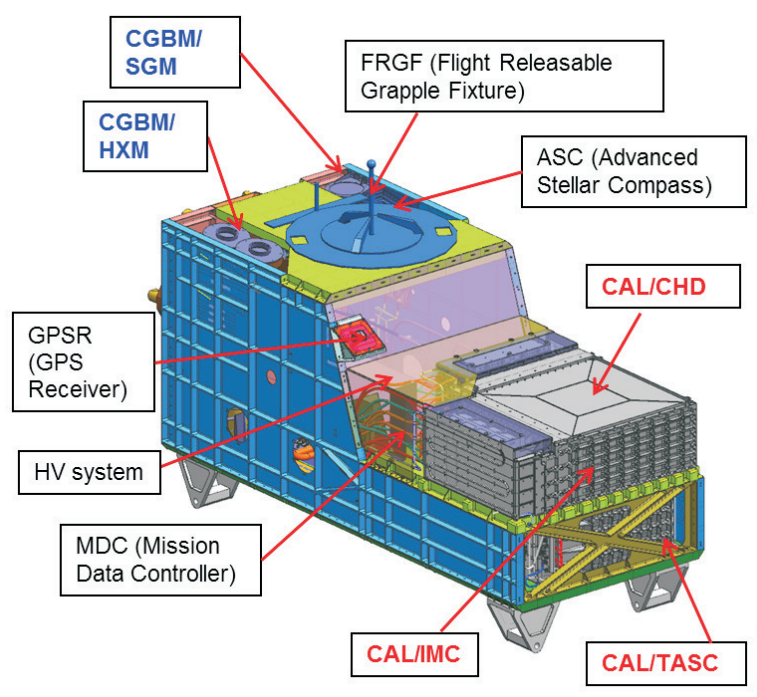

Figure 1. Drawing of the CALET payload. On the ISS the payload will be installed in such a way to have open sky on top, Earth on bottom.

\subsection{CALET-CAL instrument}

The CALET calorimeter (see figure 2) is composed of a charge detector (CHD), a pre-shower imaging calorimeter (IMC) and a total absorption calorimeter (TASC). It is optimized for particle identification and energy measurement of several cosmic-ray species:

- electrons and positrons in the $1 \mathrm{GeV}-20 \mathrm{TeV}$ energy range (with no charge sign discrimination);

- photons from few $\mathrm{GeV}$ to $\sim 10 \mathrm{TeV}$;

- nuclei up to the Fe region, with energy from tens of $\mathrm{GeV}$ to $\sim 1 \mathrm{PeV}$;

- ultra-heavy $(\mathrm{Z}>28)$ nuclei with $\mathrm{E}>600 \mathrm{MeV} /$ nucleon (in this case, with no energy measurement).

The CHD, positioned on top of the IMC structure, is composed of two layers of plastic scintillator with mutually orthogonal segmentation in 14 bars (SciBars), each of dimensions $3.2 \times 1.0 \times 44.8 \mathrm{~cm}^{3}$ and read by a photo-multiplier tube (PMT) and front-end circuit (FEC) with charge sensitive amplifier, for a total of 28 channels. The CHD determines the charge absolute value $|\mathrm{Z}|$ of the incoming charged particle, through the $Z^{2}$ dependence of the specific ionization loss. The very low uncertainty in the $\mathrm{Z}$ measurement [3] ( 0.1 for light nuclei up to $\mathrm{B}, 0.3$ in the Fe region) allows for resolving individual chemical elements with $\mathrm{Z}$ from 1 to 40 .

The IMC is a finely segmented sampling calorimeter, with surface area of $45 \times 45 \mathrm{~cm}^{2}$ and total thickness of 3 radiation lengths $\mathrm{X}_{0}$; internally, 8 double layers of scintillating fibres (SciFi, $1 \mathrm{~mm}^{2}$ cross-section) are interleaved with a sequence of 7 tungsten plates: 5 of thickness $0.2 \mathrm{X}_{0}$ and 2 of thickness $1.0 \mathrm{X}_{0}$. The fibres of each double layer are mutually orthogonal and arranged in belts, each read by a 64-channel multi-anode PMT (MAPMT) and VA front-end ASIC circuit, for a total of 7168 channels. The IMC fine granularity allows for precise determination of the incoming particle trajectory, localization of the starting point of the secondary shower possibly generated, discrimination 


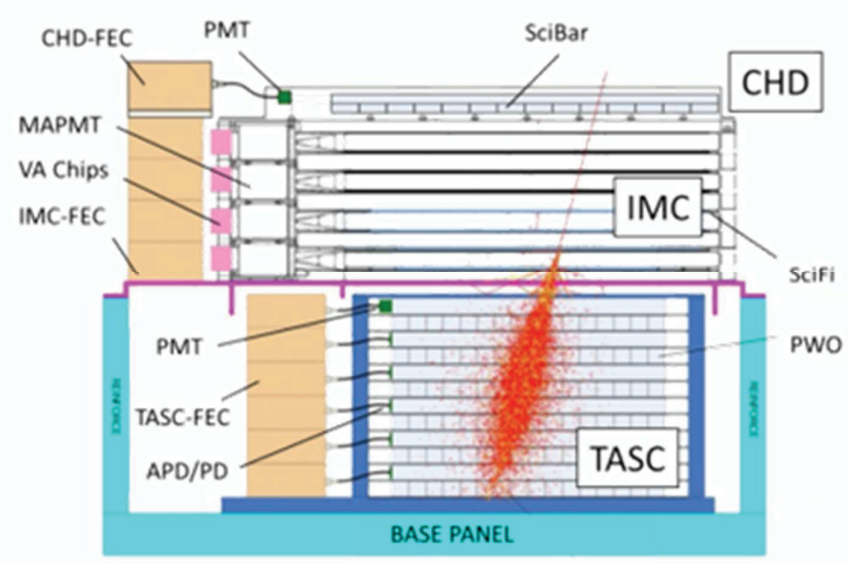

Figure 2. Drawing of the CALET-CAL instrument, with a typical shower of secondary particles generated by a $1 \mathrm{TeV}$ electron incident from top.

of the primary incident particle against possible backscattering from the shower developing in the IMC and underlying TASC.

The TASC is a homogeneus calorimeter made of 192 lead tungstate (PWO) logs with dimensions $20 \times 19 \times 320 \mathrm{~mm}^{3}$ and arranged in 12 layers, oriented along alternatively orthogonal directions. Each $\log$ in the top layer is read by a PMT, while a dual photodiode / avalanche photodiode (PD/APD) system is used for read-out of the other layers. The TASC is specifically designed to measure the energy of the incident particle with excellent resolution: $2 \%$ (3\%) for electrons (gamma-rays) with energy $\mathrm{E}>10 \mathrm{GeV}$, better than $35 \%$ for nuclei up to $\sim 100 \mathrm{TeV}$ energy.

Moreover (see e.g. [4]), by exploiting the TASC and IMC shower imaging capabilities, a proton rejection power of $\sim 1 \cdot 10^{5}$ can be achieved in the electron sample, with a selection efficiency better than $80 \%$, sufficient to keep the proton contamination below a few percent in the observation of cosmic-ray electrons in the multi-TeV region.

The total thickness of the CALET-CAL instrument is equivalent to $30 \mathrm{X}_{0}$ and 1.5 nuclear interaction lengths $\lambda_{I}$. Its effective geometrical factor is $0.12 \mathrm{~m}^{2} \mathrm{sr}$ for high-energy electrons and nuclei. The angular resolution is better than $0.3^{\circ}$ for electrons and gamma-rays in the $10 \mathrm{GeV}-1 \mathrm{TeV}$ energy range.

Prototypes of the CHD, IMC and TASC detectors were extensively tested at CERN SPS with beams of muons at 150 and $180 \mathrm{GeV}$ energy, electrons with energies from 10 to $290 \mathrm{GeV}$, protons from 30 to $400 \mathrm{GeV}$, ion fragments at 13 and $30 \mathrm{GeV} /$ nucleon. Results of the data analysis [5] demonstrate that the measured detector performances meet the design specifications.

\subsection{CGBM instrument}

The CGBM instrument [6] is dedicated to observation of gamma-ray bursts (GRB) and other X/gamma-ray transient phenomena, with a broad energy coverage from hard X-rays to soft gammarays, specifically in the range from $7 \mathrm{keV}$ to $20 \mathrm{MeV}$; these data can be correlated with simultaneous gamma-ray observations of CALET-CAL instrument in the region from few $\mathrm{GeV}$ to several $\mathrm{TeV}$. 
The CGBM consists of two hard X-ray monitor (HXM) units and one soft gamma-ray monitor (SGM) unit.

Each HXM unit contains a $\mathrm{LaBr}_{3}(\mathrm{Ce})$ scintillator crystal read by a PMT and shaped as two superposed cylinders with diameter of 6.6 and $7.9 \mathrm{~cm}$ respectively and thickness $0.6 \mathrm{~cm}$ each. The $\mathrm{LaBr}_{3}(\mathrm{Ce})$ scintillator is here used in space for the first time for celestial gamma-ray observations. The field of view is limited by a collimator within $\approx 58^{\circ}$ from the vertical axis, to reduce possible contamination from cosmic X-ray background and bright X-ray astrophysical sources.

The SGM unit contains a BGO scintillator read a by PMT and shaped as a cylinder with $10 \mathrm{~cm}$ diameter and $7.6 \mathrm{~cm}$ thickness.

The energy resolution of HXM and SGM is $\sim 3 \%$ and $\sim 15 \%$, respectively, at $662 \mathrm{keV}$.

\section{CALET science}

In the present section the main CALET science objectives are illustrated, as outlined below.

- Search for signatures of nearby astrophysical sources of cosmic rays (CR) in the multi-TeV cumulative $\mathrm{e}^{-} \mathrm{e}^{+}$energy spectrum; accurate and high-statistics spectral measurement in the $1 \mathrm{GeV}-20$ $\mathrm{TeV}$ region, searching for dark matter signatures and fine spectral features. See Sect. 3.1.

- Search for dark matter signatures in the $10 \mathrm{GeV}-10 \mathrm{TeV}$ gamma-ray spectrum; accurate spectral measurement of gamma-ray galactic/extragalactic background radiation and bright astrophysical sources. See Sect. 3.2.

- Study of CR acceleration and propagation mechanisms, with the measurement of nuclei spectra from $\mathrm{H}$ to the $\mathrm{Fe}$ region for energies from tens of $\mathrm{GeV}$ up to several $\mathrm{TeV}$, with the measurement of the $\mathrm{B} / \mathrm{C}$ flux ratio up to several $\mathrm{TeV} /$ nucleon and with the determination of abundances of ultraheavy nuclei up to $Z=40$. See Sect. 3.3.

Other important science items, not specifically treated here, are listed below.

- Detection and study of X-/gamma-ray transients in the energy region $7 \mathrm{keV}-20 \mathrm{MeV}$ with CGBM.

- Study of solar modulation of the $\mathrm{e}^{-} \mathrm{e}^{+}$flux below $\sim 10 \mathrm{GeV}$.

\subsection{Cosmic-ray electrons and positrons}

Multi-TeV electrons originated in SuperNova Remnant (SNR) standard sources can be observed at Earth only if the SNR is younger than $10^{5}$ years and less than $1 \mathrm{kpc}$ from the solar system, because of radiative synchrotron and inverse Compton energy losses, proportional to the squared energy, during propagation in the Galaxy [7]. Since the number of such nearby SNR's is very limited (Vela, Monogem, Cygnus Loop and few others), the electron energy spectrum around and above $1 \mathrm{TeV}$ could exhibit spectral features and be characterized by a significant anisotropy (order of 10\%) in the arrival direction, related to the localization of the sources with respect to the galactic plane.

Among its contemporary experiments, CALET has unique capabilities for identifying such features in the multi-TeV region of cumulative $\mathrm{e}^{-} \mathrm{e}^{+}$flux, namely energy resolution, proton rejection power and angular resolution, together with a long exposure in space: for example, 300 events/yr from the Vela SNR component with $\mathrm{E}>1 \mathrm{TeV}$ are expected to be collected and characterized after applying necessary data selection cuts, thus possibly giving a result as shown in figure 3 . Besides, the CALET measurement will allow for tuning models of SNR sources, since the shape of CR electron flux in the multi-TeV range is critically affected (see e.g. [7]) by several model parameters which are still not precisely known, such as exponential cut-off energy of the accelerated spectrum, acceleration time interval, value of the diffusion coefficient. 


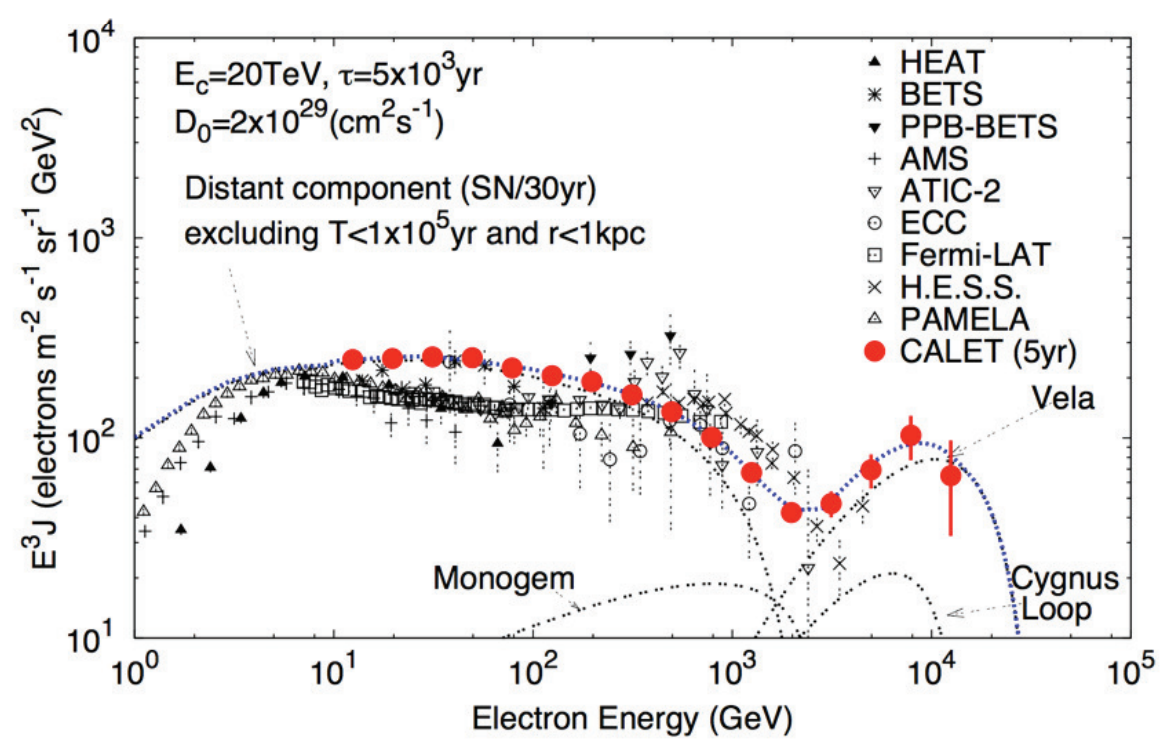

Figure 3. Expected cumulative $\mathrm{e}^{-} \mathrm{e}^{+}$energy spectrum measured with CALET after 5 years of observation, according to a possible SNR model as discussed in [7]: a distinctive signature of nearby SNR sources can be identified by CALET in the multi-TeV region. A selection of previous measurements is also reported.

Also in the sub-TeV energy region CALET accuracy and exposure will make it possible to significantly improve the knowledge of the detailed spectral shape and angular distribution of cumulative $\mathrm{e}^{-} \mathrm{e}^{+}$flux. This will help in identifying the additional unknown source, either an astrophysical object (e.g. a nearby pulsar) or annihilation/decay of dark matter particles, which has been suggested to explain recent measurements showing a clear change of slope, namely an $\mathrm{e}^{-} \mathrm{e}^{+}$flux enhancement, in the range $200 \mathrm{GeV}-1 \mathrm{TeV}$, not expected for standard SNR sources (see e.g. [8] and references therein). ${ }^{1}$

\subsection{Gamma-rays}

According to theories of annihilation or decay of dark-matter particles in the galactic halo, these phenomena should produce sharp gamma-rays lines in the sub-TeV to $\mathrm{TeV}$ energy region, superimposed on the diffuse spectrum. CALET [11] [12] will be capable of investigating such a distinctive signature, as shown e.g. in figure 4, thanks to the gamma-ray energy resolution of $3 \%$ above $100 \mathrm{GeV}$, which can be improved to $1 \%$ with a reduced $(75 \%)$ on-axis effective area, obtained by selecting only events with primary gamma-ray crossing all detector planes and at least $2 \mathrm{~cm}$ inside TASC layers (to assure complete lateral containment of the secondary shower).

CALET excellent energy resolution and good angular resolution (better than $0.4^{\circ}$, including pointing uncertainty) will allow for accurate measurements of diffuse gamma-ray emission and more than 100 bright sources at high latitude from the Fermi-LAT catalogue [13]. Given the on-axis effective area of $\approx 600 \mathrm{~cm}^{2}$ for energies above $10 \mathrm{GeV}$ (reduced by $\sim 50 \%$ at $4 \mathrm{GeV}$ ) and field of view of $\approx 45^{\circ}$ from the vertical direction, CALET is expected to detect $\sim 25000(\sim 7000)$ photons from the galactic (extra-galactic) background with $\mathrm{E}>4 \mathrm{GeV}$ and $\sim 300$ photons from the Vela pulsar with $\mathrm{E}>5 \mathrm{GeV}$.

\footnotetext{
${ }^{1}$ The presence of an additional source is also required to explain the now established rise of positron fraction in the $10 \mathrm{GeV}$ - $500 \mathrm{GeV}$ energy range (see e.g. [9], [10]).
} 


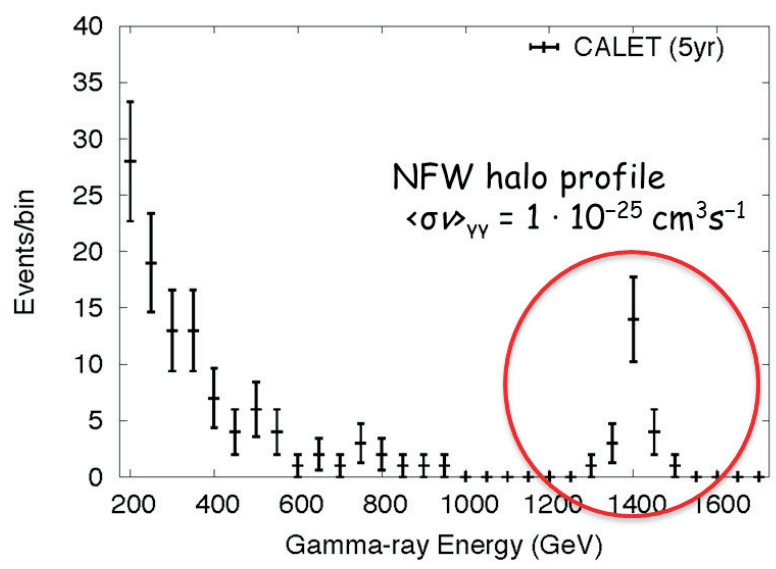

Figure 4. Expected CALET 5-years measurement of a possible $1.4 \mathrm{TeV}$ gamma-ray line from dark matter in the region of galactic centre, also including galactic diffuse background, according to [11].

\subsection{Cosmic-ray protons and nuclei}

It is still not clear to what extent the energy spectra of cosmic-ray nuclei in the $10 \mathrm{GeV}-1 \mathrm{PeV}$ region are well described by a single power law $\mathrm{E}^{-\gamma}$ with $\gamma \approx 2.7$ : in particular, PAMELA collaboration reported accurate measurements [14] clearly indicating small but significant deviations from single power law spectra for both $\mathrm{H}$ and $\mathrm{He}$ in the sub-TeV region, which still need to be compared with independent high-accuracy measurements. ${ }^{2}$ The same measurements also show a clear difference of $\approx 3 \%$ between the spectral indexes of $\mathrm{H}$ and $\mathrm{He}$ : it is therefore reasonable to investigate similar differences for other nuclei species. Finally, it is still an open question whether there is a spectral cut-off ("knee") below $1 \mathrm{PeV}$ for the various nuclei species. These open points reflect in uncertainties in the understanding of the cosmic-ray shock acceleration mechanism taking place in SNR sources. More accurate measurements of the individual energy spectra of CR nuclei, especially approaching the $\mathrm{PeV}$ energy region, are therefore needed.

CALET will be able to identify CR nuclei with individual element resolution and measure their energies in the range from few tens of $\mathrm{GeV}$ to several hundreds of $\mathrm{TeV}$. Its thickness $\left(30 \mathrm{X}_{0}, 1.5 \lambda_{I}\right)$ allows for highly efficient nuclear interaction of the incoming nucleus and subsequent good confinement of the electromagnetic core of the secondary shower, thus achieving an energy resolution better than $35 \%$ for nuclei up to $\sim 100 \mathrm{TeV}$. In 5 years of data taking on the ISS, CALET is expected to measure the proton energy spectrum up to $\sim 900 \mathrm{TeV}$ and the He spectrum up to $\sim 400 \mathrm{TeV} /$ nucleon (see figure 5) and to determine the fluxes of the more abundant heavy nuclei with good statistical precision, up to $\sim 20 \mathrm{TeV} /$ nucleon for $\mathrm{C}$ and $\mathrm{O}$ and $\sim 10 \mathrm{TeV} /$ nucleon for $\mathrm{Ne}, \mathrm{Mg}, \mathrm{Si}$ and $\mathrm{Fe}$.

Important information on the $\mathrm{CR}$ propagation in the galaxy is obtained from the secondary-toprimary flux ratio for $\mathrm{CR}$ elements, in particular the $\mathrm{B} / \mathrm{C}$ ratio [17], which is known to follow a power law in energy $\mathrm{E}^{-\delta}$ for energies below $\sim 100 \mathrm{GeV} /$ nucleon; an accurate measurement of $\delta$ and the determination whether the $\mathrm{B} / \mathrm{C}$ ratio deviates from a simple power law above $\sim 100 \mathrm{GeV} /$ nucleon are crucial for discriminating between different models of CR propagation. CALET will provide new data

\footnotetext{
${ }^{2}$ Preliminary measurements of $\mathrm{H}$ and He spectra have been published by AMS-02 collaboration [15] [16], without quantitative comparisons with PAMELA results.
} 


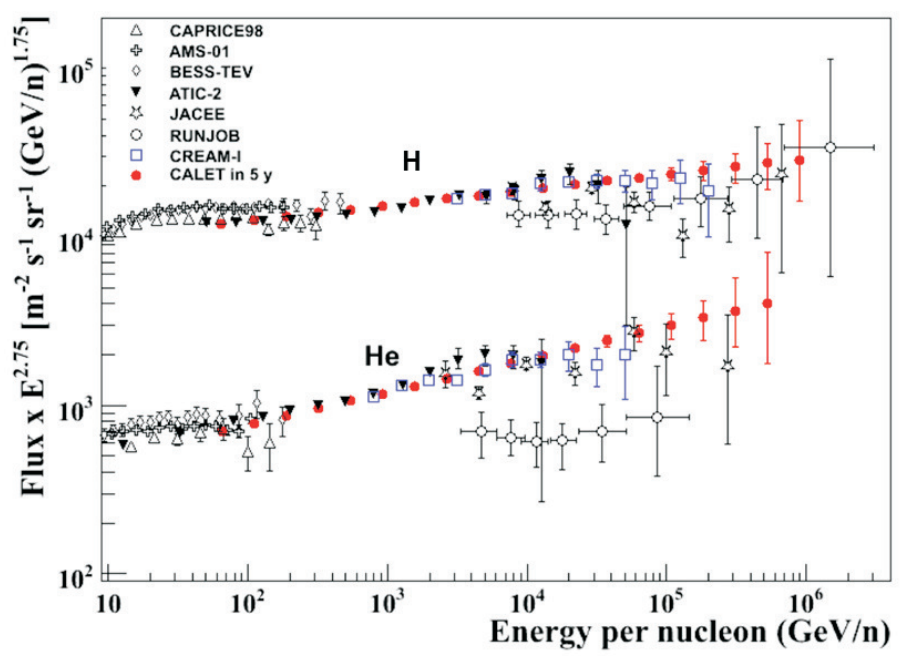

Figure 5. Expected CALET measurement of the energy spectra of $\mathrm{H}$ and He after 5 years of observation, compared with a selection of previous direct measurements.

to improve the accuracy of the present measurements above $100 \mathrm{GeV} /$ nucleon and extend them above $1 \mathrm{TeV} /$ nucleon: in 5 years, CALET can determine $\delta$ within an uncertainty of 0.05 .

Moreover [18], by exploiting the CHD particle identification capability, CALET will measure the abundances of ultra-heavy CR nuclei at few $\mathrm{GeV} /$ nucleon for $\mathrm{Z}$ up to 40 , with an expected statistics in 5 years from 2 to 4 times larger than collected by the TIGER experiment.

\section{References}

[1] S. Torii et al., Nucl. Instr. And Meth. A 630, 55-57 (2011)

[2] S. Torii et al., Proc. of $33^{\text {rd }}$ ICRC, 245, 1-4 (2013)

[3] P.S. Marrocchesi et al., Nucl. Instr. And Meth. A 659, 477-483 (2011)

[4] Y. Akaike et al., Adv. Space Res. 45, 690-697 (2010)

[5] Y. Akaike et al., Proc. of $33^{\text {rd }}$ ICRC, 726, 1-4 (2013)

[6] K. Yamaoka et al., Proc. of $33^{\text {rd }}$ ICRC, 1007, 1-4 (2013)

[7] T. Kobayashi et al., Astrophys. J. 601, 340-351 (2004)

[8] M. Ackermann et al., Phys. Rev. D 82, 092004, 1-20 (2010)

[9] O. Adriani et al., Nature 458, 607-609 (2009)

[10] L. Accardo et al., Phys. Rev. Lett. 113, 121101, 1-9 (2014)

[11] K. Yoshida et al., Proc. of $33^{\text {rd }}$ ICRC, 735, $1-4$ (2013)

[12] M. Mori et al., Proc. of $33^{\text {rd }}$ ICRC, 248, 1-4 (2013)

[13] A.A. Moiseev et al., Proc. of $33^{\text {rd }}$ ICRC, 627, 1-4 (2013)

[14] O. Adriani et al., Science 332, 6025, 69-72 (2011)

[15] S. Haino et al., Proc. of $33^{\text {rd }}$ ICRC, 1265, 1-4 (2013) 
[16] V. Choutko et al., Proc. of $33^{\text {rd }}$ ICRC, 1262, 1-4 (2013)

[17] A. Obermeier et al., Astrophys. J. 752, 69, 1-7 (2012)

[18] B.F. Rauch et al., Proc. of $33^{\text {rd }}$ ICRC, 819, 1-4 (2013) 